\title{
Analytical Model for Reluctance and Cage Rotor Bar Magnetic Gear
}

\author{
Yusuf Akcay ${ }^{1}$, Tom $\mathrm{Cox}^{2}$, Alessandro Costabeber ${ }^{3}$, Giacomo Sala ${ }^{4}$ \\ (1), (2), (3), (4) Dept. of Electrical and Electronics Engineering, Univ. of Nottingham, United Kingdom, \\ yusuf.akcay@nottingham.ac.uk.
}

\begin{abstract}
Magnetic Gearboxes are becoming more attractive in gearing area because of their high torque density and high efficiency. In this paper, we proposed a two-dimensional analytical model in a coaxial magnetic gearbox by considering design aspects which can make the design process more reliable, efficient and cost-effective. The proposed analytical model is based on the solution of Laplace's and Poisson's equations for each subdomain, i.e., magnets, air gap, slots, and bars. The global solution is obtained using boundary and continuity conditions. The analytical model can be used as a design tool for a magnetic gearbox with more design aspects compared with previous proposed methods. The analytical results of field distribution, induced bar current and electromagnetic torque are obtained and compared with finite element analysis.
\end{abstract}

Index Terms-Analytical method, cage rotor bar, magnetic gear, magnetic field, permanent magnet.

\section{INTRODUCTION}

$\mathrm{M}$ AGNETIC gearboxes give promising results by comparison with mechanical counterparts in terms of many factors, i.e., less acoustic noise, almost maintenance-free operation, high efficiency and high torque density [1]-[2].

One of the effective numerical analysis methods, finite element method (FEM), is widely used in machine design and similar fields. FEM allows accurate computation of airgap field, torque and induced eddy current in rotor bars. [3]-[6]. However, Magnetic gearboxes (MGs) possess complex structures that contain more rotational parts and couplings. That makes design process by FEM more complex and time consuming than analytical counterparts. Therefore, analytical methods are helpful tools to analyze MGs for the first step of the design process due to quick evaluation. Previously, a twodimensional (2D) analytical model based on the analysis of subdomains has been proposed by Thierry et al. [7]. The model is proposed, illustrating a coaxial magnetic gearbox with radially magnetized PMs for an exact analytical solution of the magnetic field distribution and electromagnetic torque. The method is helpful to design a coaxial magnetic gear as a first step of design process. In [8], a 2D analytical method to calculate the transmitted torque and torque ripple has been proposed for MGs considering permanent magnets with halbach array layouts. The proposed method allows comparison of the performance of MGs with different magnetized PMs. It is well known that the halbach array in PMs gives stronger fields in airgap. In [9] a coaxial MG with halbach array is analyzed with a similar 2D analytical method. In the paper, the effects of the width and height of modulating piece and thickness of outer rotor yoke are investigated. Another similar approach for a 2D analytical method has been introduced for a new type of MG [10]. The proposed method calculates the magnetic field distribution in axial-field MGs. In [11], a similar approach has been proposed for a coaxial MG for transmitted torque and airgap flux field calculation, where, ferromagnetic poles are replaced with superconductive material. The method proposed in [12] allows to calculate the average torque and flux components. In [13], detailed analyses are done by a $2 \mathrm{D}$ analytical method. In the analyses, the influences of the number of pole-pairs, the magnetization patterns (i.e. radial, parallel, ideal halbach and two segment halbach) the size of slot-opening and the segment ratio of the two-segment halbach pattern on the transmitted torque and unbalanced magnetic forces have been analytically investigated and compared with the FEA results. A comparison of the computation time between the 2-D analytical method, the optimized 2-D analytical method and FEA is done in [14]. The comparison shows a significant difference between the analytical method and FEA. In fact, the computation time with the analytical method is highly dependent on the number of harmonics selected for the solution of each region. However, unless a very high-number of pole-pairs selected, it is still significantly faster with the analytical method than FEA.

Although the aforementioned methods are useful to evaluate MGs, they only evaluate MGs within limited design aspects such as the geometry of ferromagnetic poles and magnetization pattern of the PMs. The methods are not applicable to evaluate effects of pole-arc to pole-pitch ratio of PMs, reluctance effects created by the salient structure of inner PM poles and addition of cage rotor bars in the inner rotor of MGs. The idea of adding cage rotor bars is firstly mentioned in [15]. The paper investigates the effects of the rotor bars under overload conditions. The resulting proposed gear improves torque performance at overload conditions.

In this paper, an accurate analytical method is suggested by considering the effects of pole-arc to pole-pitch ratio on the inner rotor PMs, saliency due to shape of the inner rotor in which reluctance torque is generated and lastly effects of 
adding cage rotor bars in the inner rotor to improve overload torque transmission capability are analyzed.

The suggested analytical method can address more design aspects and provides better evaluation of MGs. This paper first will give the method for conventional gearbox then will focus on the reluctance effects and lastly will consider cage rotor bar to improve design performance at overload conditions. Airgap flux and electromagnetic torque are compared. Finally, the evaluation of the eddy current in the rotor bar is analysed in depth, being important in order to calculate the torque produced by the inner rotor of the considered layout of magnetic gearbox.

\section{MOTOR GEOMETRY AND ASSUMPTIONS}

The configuration of the cage rotor MG is shown in Fig.1. In this paper, three different topologies are used to evaluate the proposed analytical method and to compare the results with those obtained from FEA. The parameters of the MGs are given in table I. The inner rotor is modified to investigate the reluctance effect and cage rotor bars. The outer permanent magnets are considered stationary, while the modulating ring and the inner permanent magnets are both rotational, and are designed as the outer and inner rotors, respectively. The rotor bars are equally placed in the inner rotor. The 6-bar MG has bars 60 degrees apart, placed between adjacent PMs, while the 12-bar MG has bars 30 degrees apart.

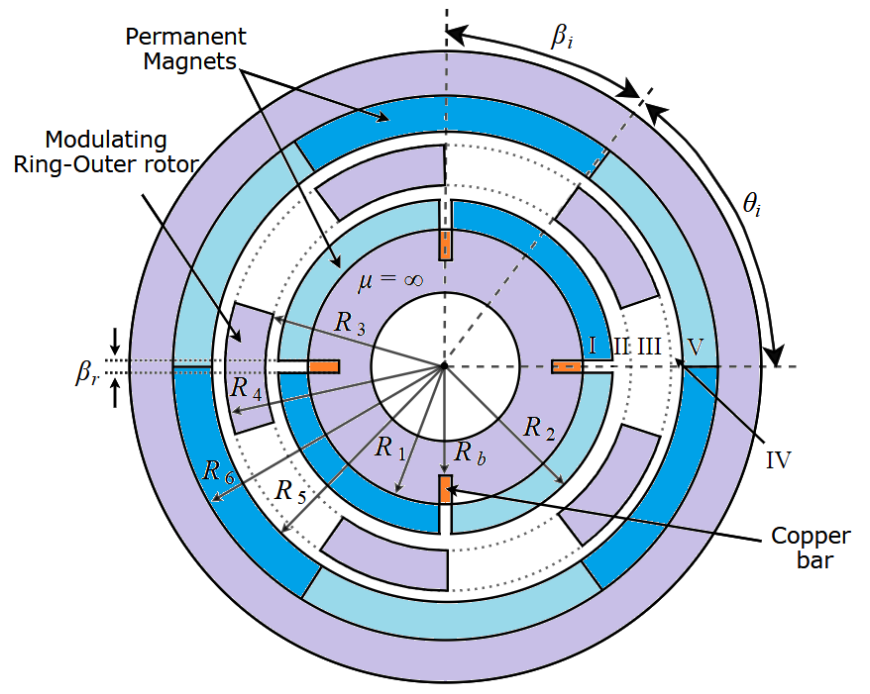

Fig. 1. Proposed MG topology with cage rotor bars

The analytical model is given in this section for all topologies. The following assumption has been made.

1. End effects are neglected

2. Outer back iron, modulating pieces and inner back iron have infinite permeability

3. PMs have constant permeability and remanent flux density

4. The angular velocity of the rotor is known and constant.
5. The electrical conductivity of the rotor bars is constant.

\section{ANALYTICAL SOLUTION}

In the analysis, the field is calculated by using magnetic vector potential. The models investigated in the paper are represented by the following regions of interest:

1. Rotor bar (b)

2. Inner slot (r)

3. Inner magnet (I) and outer magnet (V)

4. Inner airgap (II) and outer airgap (IV)

5. Ferromagnetic pole (III)

The letters represent the notation used for each region.

\section{A. Magnetization of Permanent Magnet}

The PMs in the analytical models are represented with Fourier series expansion of their magnetization. In this paper, radial magnetization is assumed. The different type of magnetization can be found in [13]. The magnetization vector can be represented by the following expression:

$$
\vec{M}=\frac{\overrightarrow{B_{r}}}{\mu_{0}}
$$

where $\mu_{0}$ is permeability of free space and $B_{r}$ is the remanent magnetization of the PMs. $\vec{M}$ is represented in polar coordinate as follows:

$$
\vec{M}=M_{r} \cdot \vec{r}+M_{\theta} \cdot \vec{\theta}
$$

where $\vec{r}$ and $\vec{\theta}$ denote the direction vectors in radial and tangential directions. $M_{r}$ and $M_{\theta}$ are the magnitudes of radial and tangential components of $\vec{M}$, respectively. The Fourier series expressions for these components are

$$
\begin{array}{ll}
M_{r}=\sum_{n=1,3,5 \ldots}^{\infty} M_{r n} \cdot \cos \left(n p\left(\theta-\varphi_{I, O}\right)\right) & \forall n \\
M_{\theta}=\sum_{n=1,3,5 \ldots}^{\infty} M_{\theta n} \cdot \sin \left(n p\left(\theta-\varphi_{I, O}\right)\right) & \forall n
\end{array}
$$

where $M_{r n}$ and $M_{\theta n}$ denote the $n t h$ harmonic element of the radial and tangential components, $\theta$ is the magnetic polepairs angular displacement, $\varphi_{I}$ and $\varphi_{O}$ are the initial angle of the outer stationary magnetic pole-pairs and the inner rotor, respectively. Different types of magnetization affect the field distribution in the motor. However, they do not change the procedure for magnetic field calculation. For radially magnetized magnets, $M_{r n}$ and $M_{\theta n}$ are mathematically expressed as given as follows:

$$
\begin{gathered}
M_{r n}=\frac{4}{\mu_{0}} \frac{B_{r}}{n a_{m} \pi} \sin \left\{\frac{n \alpha_{m} \pi}{2}\right\} \\
M_{\theta n}=0
\end{gathered}
$$

where $\alpha_{m}$ is the magnet angular width. The magnetization pattern of the radially magnetized outer permanent magnets is shown in Fig. 2. 


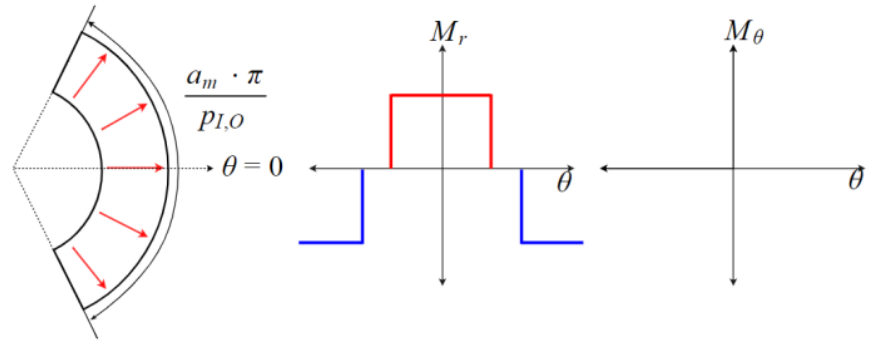

Fig. 2. Radially magnetized PMs

where $p_{I}$ and $p_{O}$ are the number of pole pairs in the inner rotor and the outer stationary part.

\section{B. Inner Rotor Reference Frame}

Due to the presence of bars in the inner rotor, the model is developed in the reference frame of the inner rotor. The primary source of magnetomotive force in the model is given by the PMs. The permanent magnet excitation is given in (2). Considering for the reference frame of the inner rotor, (2) can be rewritten as follows:

$$
\begin{aligned}
M(r, \theta, t) & =M_{r n} \cos \left(s p_{I, O}\left(\theta-\varphi_{I, O}\right)-\omega_{r n} t\right) \cdot \vec{r} \\
& +M_{\theta n} \sin \left(s p_{I, O}\left(\theta-\varphi_{I, O}\right)-\omega_{r n} t\right) \cdot \vec{\theta}
\end{aligned}
$$

The synchronous speed of the magnetic field produced by the PMs changes with the space harmonics. The angular velocity of the $n$th rotating magnetic field $\omega_{r n}$ is given by

$$
\begin{aligned}
& \omega_{s n}=\left(N_{o} \frac{2 \pi}{60} n\right) \\
& \omega_{r n}=\left(p_{o} s \omega_{s n}\right)
\end{aligned}
$$

where $\omega_{s n}$ is the stator angular speed, $\omega_{r m}$ is the rotor angular speed $n$ is the harmonic component, $N_{o}$ is the outer PM rotational speed in revolution per minute $(\mathrm{rpm})$, and $s$ is the rotor slip.

\section{Magnetic Field Calculation}

The magnetic vector potential in each region is governed by several equations, Poisson's, Laplace's and complex Helmholtz equations. The PM region is solved by Poisson's equation:

$$
\frac{\partial^{2} A_{I, V}}{\partial r^{2}}+\frac{1}{r} \frac{\partial A_{I, V}}{\partial r}+\frac{1}{r^{2}} \frac{\partial^{2} A_{I, V}}{\partial \theta^{2}}=\frac{\mu_{0}}{r} \frac{\partial M_{r}}{\partial \theta}
$$

where the $A_{I}$ and $A_{V}$ vector potential for inner and outer magnet region, $M_{r}$ is the magnetization of PMs. The airgap regions r, II, III and IV are governed by Laplace's equation as:

$$
\frac{\partial^{2} A_{r, I I, I I I, I V}}{\partial r^{2}}+\frac{1}{r} \frac{\partial A_{r, I I, I I I, I V}}{\partial r}+\frac{1}{r^{2}} \frac{\partial^{2} A_{r, I I, I I I, I V}}{\partial \theta^{2}}=0
$$

where $A_{I I}$ and $A_{I V}$ are vector potential for inner and outer airgap, respectively. $A_{I I I}$ is vector potential for the ferromagnetic pole-pieces and $A_{r}$ is for the inner reluctance slot. The cage rotor bar area is solved by the complex
Helmholtz equation, related to the main field contributions of nth order, as follows:

$$
\frac{\partial^{2} A_{b}}{\partial r^{2}}+\frac{1}{r} \frac{\partial A_{b}}{\partial r}+\frac{1}{r^{2}} \frac{\partial^{2} A_{b}}{\partial \theta^{2}}=j \omega_{r n} \sigma \mu_{0} A_{b}
$$

where $A_{b}$ is vector potential for cage rotor bar.

\section{Potential Function}

As explained in Section II, magnetic vector potentials for various regions will have only $z$-component. For the analysis of the cage effect, vector potential in the inner rotor reference frame can be represented for each region as follows:

$$
\begin{aligned}
& \overline{A_{b}}(r, \theta, t)=\operatorname{Re}\left\{A_{b}(r, \theta) e^{j \omega_{m} t}\right\} \\
& \overline{A_{I}}(r, \theta, t)=\operatorname{Re}\left\{A_{I}(r, \theta) e^{j \omega_{m} t}\right\} \\
& \overline{A_{I I}}(r, \theta, t)=\operatorname{Re}\left\{A_{I I}(r, \theta) e^{j \omega_{m} t}\right\} \\
& \overline{A_{I I I}}(r, \theta, t)=\operatorname{Re}\left\{A_{I I I}(r, \theta) e^{j \omega_{m} t}\right\} \\
& \overline{A_{I V}}(r, \theta, t)=\operatorname{Re}\left\{A_{I V}(r, \theta) e^{j \omega_{m} t}\right\} \\
& \overline{A_{V}}(r, \theta, t)=\operatorname{Re}\left\{A_{V}(r, \theta) e^{j \omega_{m} t}\right\}
\end{aligned}
$$

where Re represents the real part of the complex function. Similarly, the magnet excitation is also represented with complex notations as given in (14)

$$
\begin{aligned}
& \vec{M}(r, \theta, t)=\operatorname{Re}\left\{\vec{M}(r, \theta) e^{j \omega_{m} t}\right\} \\
& \vec{M}(r, \theta)=M_{r n} e^{-j n P_{1, o}(\theta-\delta)} \cdot \vec{r}+M_{\theta n} e^{-j\left(n P_{1, o}(\theta-\delta)-\pi / 2\right)} \cdot \vec{\theta}
\end{aligned}
$$

The magnetic vector potential for the inner and outer air gaps is given by

$$
\begin{aligned}
A_{I I}(r, \theta)=\sum_{n=1}^{\infty} & \left(A_{I I, n} r^{n}+B_{I I, n} r^{-n}\right) \cos (n \theta) \\
& +\sum_{n=1}^{\infty}\left(C_{I I, n} r^{n}+D_{I I, n} r^{-n}\right) \sin (n \theta) \\
A_{I V}(r, \theta)=\sum_{n=1}^{\infty}( & \left.A_{I V, n} r^{n}+B_{I V, n} r^{-n}\right) \cos (n \theta) \\
& +\sum_{n=1}^{\infty}\left(C_{I V, n} r^{n}+D_{I V, n} r^{-n}\right) \sin (n \theta)
\end{aligned}
$$

where $A_{I I, n}, B_{I I, n}, C_{I I, n}, D_{I I, n}, A_{I V, n}, B_{I V, n}, C_{I V, n}, \quad$ and $D_{I V, n}$ are the constants to be obtained. Magnetic vector potential in the PM regions is given by

$$
\begin{aligned}
A_{I}(r, \theta) & =\sum_{n=1}^{\infty}\left(A_{I, n} r^{n}+B_{I, n} r^{-n}+F_{I, n}(r) \cos \left(n \varphi_{I}\right)\right) \cos (n \theta) \\
& +\sum_{n=1}^{\infty}\left(C_{I, n} r^{n}+D_{I, n} r^{-n}+F_{I, n}(r) \sin \left(n \varphi_{I}\right)\right) \sin (n \theta)
\end{aligned}
$$

where $A_{I, n}, B_{I, n}, C_{I, n}$, and $D_{I, n}$ are constants and $\varphi_{I}$ is the initial angle of the inner rotor. $F_{I, n}(r)$ is given by 


$$
F_{I, n}(r)=\left[\begin{array}{ll}
4 B_{r_{I}} p_{I} r /\left(\pi\left(1-n^{2}\right)\right) & \text { if } n=s p_{I}, s=1,3,5, \ldots \\
2 B_{r_{I}} r \ln r / \pi & \text { else if } n=p_{I}=1 \\
0 & \text { otherwise }
\end{array}\right.
$$

and in the outer magnet subdomain

$$
\begin{aligned}
A_{V}(r, \theta) & =\sum_{n=1}^{\infty}\left(A_{V, n} r^{n}+B_{V, n} r^{-n}+W_{V, n}(r) \cos \left(n \varphi_{O}\right)\right) \cos (n \theta) \\
& +\sum_{n=1}^{\infty}\left(C_{V, n} r^{n}+D_{V, n} r^{-n}+W_{V, n}(r) \sin \left(n \varphi_{O}\right)\right) \sin (n \theta)
\end{aligned}
$$

where $A_{V, n}, B_{V, n}, C_{V, n}$ and $D_{V, n}$ are constants and $\varphi_{O}$ is the initial angle of the outer rotor, which is assumed as the stationery part for the analysis. $F_{O, n}(r)$ is given by

$$
F_{V, n}(r)=\left[\begin{array}{ll}
4 B_{r_{o}} p_{O} r /\left(\pi\left(1-n^{2}\right)\right) & \text { if } n=s p_{O}, s=1,3,5, \ldots \\
2 B_{r_{o}} r \ln r / \pi & \text { else if } n=p_{O}=1 \\
0 & \text { otherwise }
\end{array}\right.
$$

The general solution of the magnetic vector potential in the ith and the rth inner slot subdomains are (19) and (20) respectively.

$$
\begin{aligned}
A_{I I I}^{i}(r, \theta)=A_{I I I, 0}^{i}+B_{I I I, 0}^{i} & \ln r+\sum_{n=1}^{\infty}\left(A_{I I, k}^{i}\left(\frac{r}{R_{4}}\right)^{\frac{k \pi}{\beta_{i}}}\right. \\
& \left.+B_{I I I, k}^{i}\left(\frac{r}{R_{3}}\right)^{-\frac{-k \pi}{\beta_{i}}}\right) \cos \left(\frac{k \pi}{\beta_{i}}\left(\theta-\varphi_{i}\right)\right) \\
A_{r}^{r}(r, \theta)=A_{r, 0}^{r}+B_{r, 0}^{r} & \ln r+\sum_{n=1}^{\infty}\left(A_{r, z}^{r}(r)^{\frac{k \pi}{\beta_{r}}}\right. \\
& \left.+B_{r, z}^{r}(r)^{-\frac{k \pi}{\beta_{r}}}\right) \cos \left(\frac{k \pi}{\beta_{r}}\left(\theta-\varphi_{r}\right)\right)
\end{aligned}
$$

where $A_{I I I, 0}^{i}, B_{I I I, 0}^{i}, A_{I I I, k}^{i}, B_{I I I, k}^{i}, A_{r, 0}^{r}, B_{r, 0}^{r}, A_{r, z}^{r}$ and $B_{r, z}^{r}$ are constants. The angles $\varphi_{i}$ and $\varphi_{r}$ represent the positions of the inner $r$ th slot and $i$ th ferromagnetic pole, respectively. $\beta_{i}$ and $\beta_{r}$ are the slot opening angles for the $r$ th inner slot and ith ferromagnetic pole.

The general solution in the $b t h$ bar subdomain is given by

$$
\begin{aligned}
A_{b}(r, \theta)=A_{0}^{b} J_{0}(\alpha r) & +B_{0}^{b} Y_{0}(\alpha r)+\sum_{k=1}^{\infty}\left(A_{k}^{b} J_{k \pi / \beta}(\alpha r)\right. \\
& \left.+B_{k}^{b} Y_{k \pi / \beta}(\alpha r)\right) \cos \left(\frac{k \pi}{\beta_{b}}\left(\theta-\varphi_{b}\right)\right)
\end{aligned}
$$

where $A_{0}^{b}, B_{0}^{b}, A_{k}^{b}$ and $B_{k}^{b}$ are constants, $\varphi_{b}$ and $\beta_{b}$ represent the initial positions of the rotor bars and the slot openings, respectively. The detailed analysis for the bar region is given in [3].

\section{E. Boundary Conditions}

In order to determine all the constants in the potential functions for the various regions, boundary conditions are defined. Each rotor bar on its sides and at its bottom is covered with an iron core of infinite permeability, as shown in Fig.3. Thus, the tangential component of the magnetic field at these boundaries is zero and is mathematically given as

$$
\begin{gathered}
\left.\frac{\partial A_{b}}{\partial \theta}\right|_{\theta=\theta_{b}}=0 \quad \text { and }\left.\quad \frac{\partial A_{b}}{\partial \theta}\right|_{\theta=\theta_{b}+\beta}=0 \\
\left.\frac{\partial A_{b}}{\partial r}\right|_{r=R_{b}}=0
\end{gathered}
$$

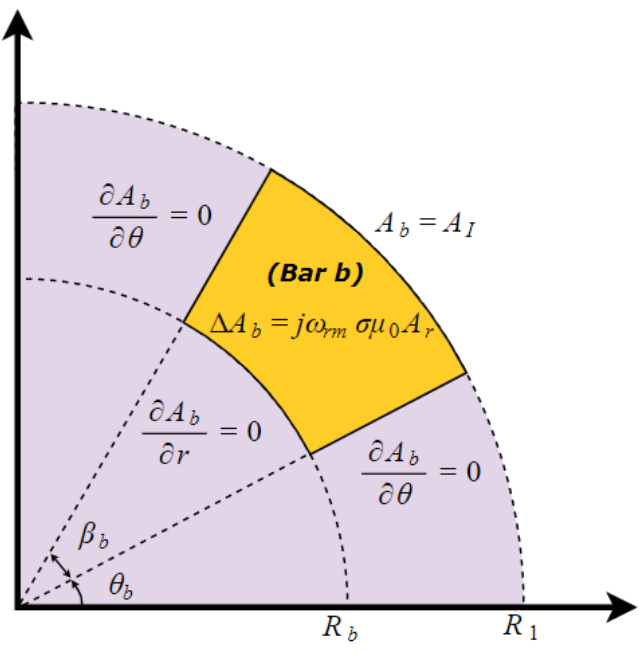

Fig. 3. Rotor bar subdomain

Similarly, each inner slot subdomain on its sides and its bottom is covered with iron core of infinite permeability, as shown in Fig.4. Therefore, the tangential component of the magnetic field at boundaries is zero and given as

$$
\begin{gathered}
\left.\frac{\partial A_{r}}{\partial \theta}\right|_{\theta=\theta_{r}}=0 \quad \text { and }\left.\quad \frac{\partial A_{r}}{\partial \theta}\right|_{\theta=\theta_{r}+\beta_{r}}=0 \\
\left.\frac{\partial A_{r}}{\partial r}\right|_{r=R_{1}}=0
\end{gathered}
$$

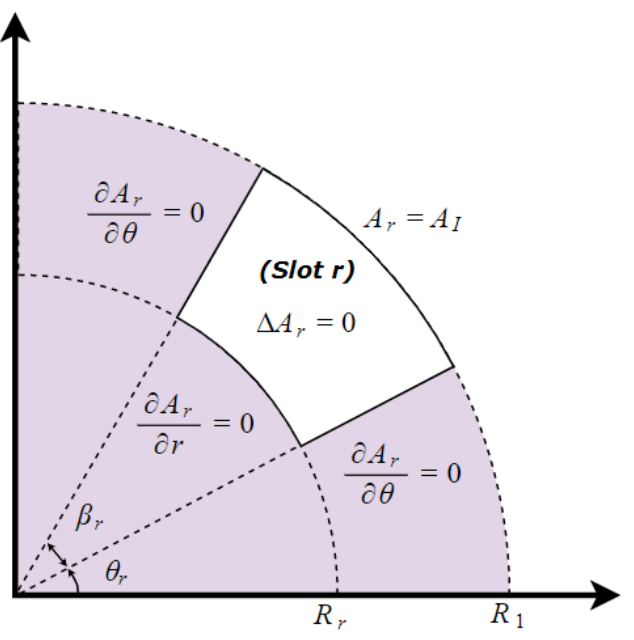

Fig. 4. Inner slot subdomai 
The continuity between the rth inner slot and the inner magnet leads to

$$
A_{r}\left(R_{1}, \theta\right)=A_{I}\left(R_{1}, \theta\right)
$$

The boundary conditions for the top of the slots and the rotor bars subdomains are given as (27) and (28), respectively.

$$
\left.\frac{\partial A_{I}}{\partial r}\right|_{r=R_{1}}=f(\theta)
$$

where

$$
\begin{array}{ll}
f(\theta)=\left.\frac{\partial A_{r}}{\partial r}\right|_{r=R_{1}} & \text { for } \theta_{r} \leq \theta \leq \theta_{r}+\beta_{r} \\
f(\theta)=0 & \text { elsewhere }
\end{array}
$$

and

$$
\left.\frac{\partial A_{I}}{\partial r}\right|_{r=R_{1}}=g(\theta)
$$

where

$$
\begin{array}{ll}
g(\theta)=\left.\frac{\partial A_{b}}{\partial r}\right|_{r=R_{1}} & \text { for } \theta_{b} \leq \theta \leq \theta_{b}+\beta_{b} \\
g(\theta)=0 & \text { elsewhere }
\end{array}
$$

\section{F. Flux Density Distribution}

The radial and tangential components of the magnetic flux density vector in the inner and outer air gaps are calculated by substituting the magnetic vector potential in region II and IV. The radial and tangential components of flux density are given by the following equations.

$$
\begin{gathered}
B_{I I, I V r}(r, \theta)=\frac{1}{r} \frac{\partial A_{I I, I V}}{\partial \theta} \\
B_{I I, I V \theta}(r, \theta)=-\frac{\partial A_{I I, I V}}{\partial r}
\end{gathered}
$$

By using (29) and (30), the following equations are obtained.

$$
\begin{aligned}
B_{I I, I V r}(r, \theta) & =\sum_{n=1}^{\infty}-\left(A_{I I, I V} \frac{n r^{n}}{r}+B_{I I, I V} \frac{n r^{-n}}{r}\right) \sin (n \theta) \\
& +\sum_{n=1}^{\infty}\left(C_{I I, I V} \frac{n r^{n}}{r}+D_{I I, I V} \frac{n r^{-n}}{r}\right) \cos (n \theta) \\
B_{I I, I V \theta}(r, \theta) & =\sum_{n=1}^{\infty}-\left(A_{I I, I V} \frac{n r^{n}}{r}-B_{I I, I V} \frac{n r^{-n}}{r}\right) \cos (n \theta) \\
+ & \sum_{n=1}^{\infty}-\left(C_{I I, I V} \frac{n r^{n}}{r}-D_{I I, I V} \frac{n r^{-n}}{r}\right) \sin (n \theta)
\end{aligned}
$$

where $B_{I I, I V r}$ and $B_{I I, I V \theta}$ are the radial and tangential components of the flux density.

\section{G. Current Density and Current in Rotor Bar}

The current value induced in the rotor bars is calculated by the current density equation given in (33).

$$
J_{b}(r, \theta)=-j \omega_{r m} \sigma A_{b}(r, \theta)
$$

The induced current in the $b t h$ rotor bar is determined by

$$
I_{b}=\int_{R_{b}}^{R_{1}} \int_{\theta_{b}}^{\beta_{b}+\theta_{b}} J_{b}(r, \theta) r d r d \theta
$$

The final equation to calculate the induced current in each rotor bar is expressed by

$$
I_{b}=\frac{-j A_{0}^{b} \sigma \omega_{r n} \beta_{b} R_{1}}{\alpha}\left(\frac{J_{1}\left(\alpha R_{b}\right) Y_{1}\left(\alpha R_{1}\right)-J_{1}\left(\alpha R_{1}\right) Y_{1}\left(\alpha R_{b}\right)}{J_{0}\left(\alpha R_{b}\right) Y_{1}\left(\alpha R_{1}\right)-J_{1}\left(\alpha R_{1}\right) Y_{0}\left(\alpha R_{b}\right)}\right)
$$

where $\sigma$ is the conductivity of the rotor bar, and $\alpha^{2}=-j \sigma \omega_{r m} \mu_{0}$. Finally, $J$ and $Y$ are the Bessel functions of the first kind and the second kind, respectively.

\section{H. Torque Transmission}

The transmitted torque of magnetic gears is one of the most important quantities, to investigate the capability of the system. Having calculated the radial and tangential components of the magnetic flux density in the inner and outer air gaps, the torque of the inner and outer rotors is computed by using the Maxwell stress tensor,

$$
\begin{gathered}
T_{e}=\frac{L R_{e}^{2}}{2 \mu_{0}} \int_{0}^{2 \pi} \operatorname{Re}\left[B_{I I r}\left(R_{e}, \theta\right) B_{I I \theta}^{*}\left(R_{e}, \theta\right)\right] d \theta \\
T_{e}=\frac{\pi L R_{e}^{2}}{\mu_{0}} \sum_{n=1}^{\infty}\left(W_{n} X_{n}^{*}+Y_{n} Z_{n}^{*}\right)
\end{gathered}
$$

with

$$
\begin{aligned}
& W_{n}=-\left(A_{I I} \frac{n r^{n}}{r}+B_{I I} \frac{n r^{-n}}{r}\right) \\
& X_{n}=-\left(C_{I I} \frac{n r^{n}}{r}-D_{I I} \frac{n r^{-n}}{r}\right) \\
& Y_{n}=C_{I I} \frac{n r^{n}}{r}+D_{I I} \frac{n r^{-n}}{r} \\
& Z_{n}=-\left(A_{I I} \frac{n r^{n}}{r}-B_{I I} \frac{n r^{-n}}{r}\right) \\
& X_{n}^{*} \text { and } Z_{n}^{*} \text { are the complex conjugate of } X_{n} \text { and } Z_{n},
\end{aligned}
$$
respectively. 


\section{RESULTS}

To evaluate the magnetic vector potential, three different gearboxes, with the parameters listed in Table I, are employed. The first model is an already existing model, conventional gearbox, the second model is a reluctance gearbox, the third model is a magnetic gearbox with cage rotor bars. Each model has its own analytical model, whereas they have partly the same subdomains. The analytical results of the conventional gearbox are not analyzed separately as the analyses are done in [7]. However, some result are used for comparison in this study.

In this section, first, the gearbox with inner reluctance slot is analyzed to predict the reluctance torque. Then, the eddy current distribution in the cage rotor bar is evaluated. The results are compared with FEA. Fig. 5 shows the parts of the three different gearbox topologies; the conventional MG, the reluctance $\mathrm{MG}$, and the cage rotor $\mathrm{MG}$.
TABLE I

DESIGN PARAMETERS

\begin{tabular}{|c|c|c|}
\hline Symbol & Parameters & Quantity \\
\hline$R 1$ & Inner magnet inner radius & $6 \mathrm{~cm}$ \\
\hline$R 2$ & Inner magnet outer radius & $6.1 \mathrm{~cm}$ \\
\hline$R 3$ & Pole pieces inner radius & $6.15 \mathrm{~cm}$ \\
\hline$R 4$ & Pole pieces outer radius & $6.65 \mathrm{~cm}$ \\
\hline$R 6$ & Outer magnet inner radius & $6.7 \mathrm{~cm}$ \\
\hline$L m$ & Outer magnet outer radius & $6.8 \mathrm{~cm}$ \\
\hline$L a$ & Modulating piece length & $0.5 \mathrm{~cm}$ \\
\hline$p_{I}$ & Axial length (active part) & $10 \mathrm{~cm}$ \\
\hline$p_{o}$ & Inner magnet pole-pairs & 3 \\
\hline$Q$ & Outer magnet pole-pairs & 13 \\
\hline$B_{r_{I}}, B_{r_{O}}$ & Remanence magnetization & $1.29 \mathrm{~T}$ \\
\hline$\omega_{s h}$ & Inner rotor angular speed & $102 \mathrm{rad} / \mathrm{s}$ \\
\hline$\sigma$ & Conductivity & $57.10^{6} \mathrm{~S} / \mathrm{m}$ \\
\hline$G_{r}$ & Gear Ratio & 5.33 \\
\hline
\end{tabular}

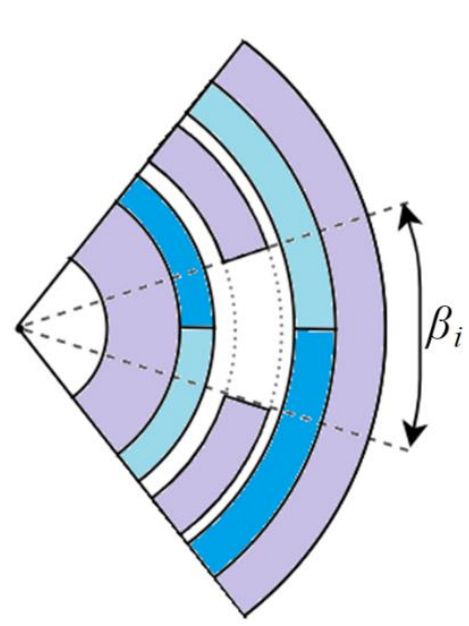

(a)

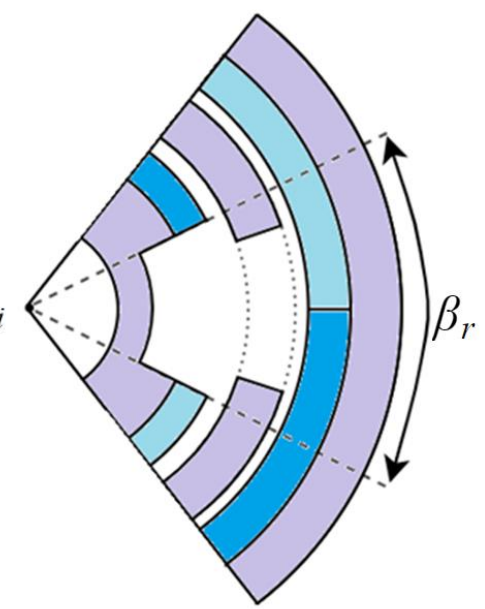

(b)

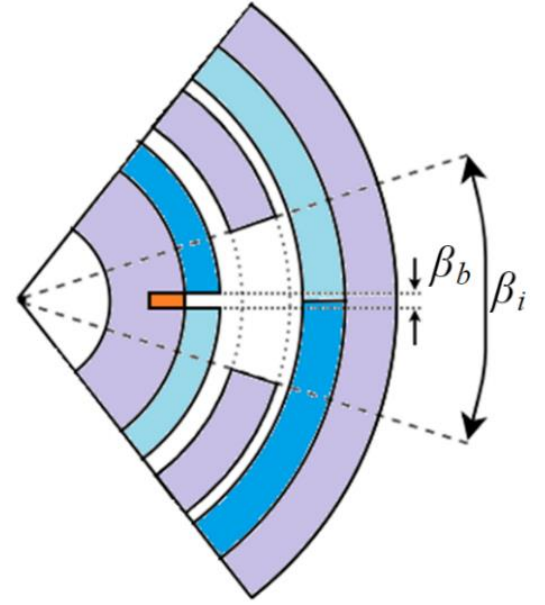

(c)

Fig. 5. Gearbox configuration, (a) conventional MG, (b) reluctance MG, (c) Cage rotor MG

\section{A. Reluctance Magnetic Gear}

The purpose of adding additional subdomains in the inner rotor is to observe the reluctance torque produced by the saliency. The analytical model of the proposed gear allows a trade-off analysis between magnet mass and overall torque. Fig. 6 shows radial and tangential components of the flux density distribution in the inner airgap, both analytical and FEM. Similarly, Fig. 7 compares the static torque values, for the different angular positions, with the values obtained for a conventional gearbox. The parameters of the reluctance magnetic gear are given in Table I. The magnet span is reduced 5 percent and the length of the inner slot is $1 \mathrm{~cm}$ for the initial analysis. The reluctance effect can be observed when the inner slot subdomain is added. However, the reluctance torque is very low when the saliency ratio is almost 1 . In Fig. 7, the ratio of the pole-arc to pole-pitch is changed between 0.9 and 0.5 . The reason for changing the pole-arc to pole-pitch ratio is to see the reluctance torque clearly. The reluctance torque production is highly affected by the geometry of the inner rotor. The length of the inner rotor slot is kept to $1 \mathrm{~cm}$ and the pole-arc to polepitch ratio is changed. The effects of the saliency on torque production is displayed in Fig. 8.

There is considerable amount of reluctance torque production which may affect the overall performance of any MGs. The static torque on the outer rotor is shown in Fig.7. The static torque is produced by the saliency created on the inner rotor and the magnetic torque. In order to highlight the pure reluctance torque, inner magnets are removed, and the results are presented, in Fig. 8, with with only outer PMs, ferromagnetic pole-pairs and inner rotor with pure ferromagnetic material. It is clear that the reluctance torque 


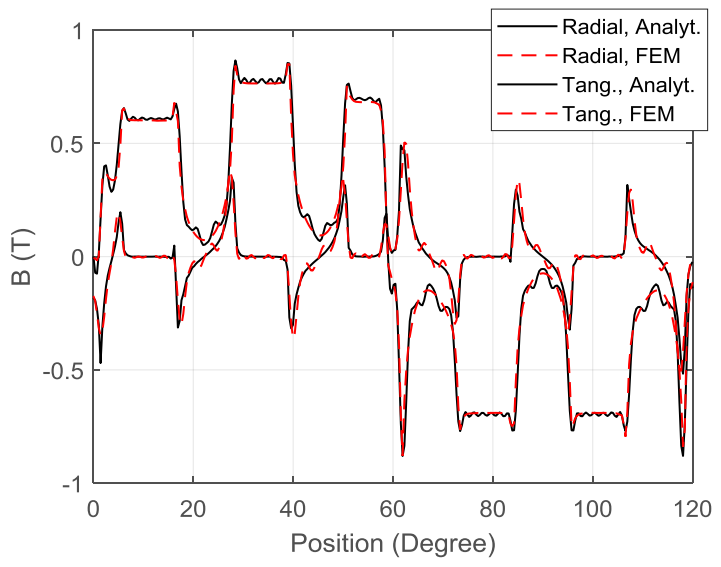

Fig. 6. Components of flux density, radial and tangential

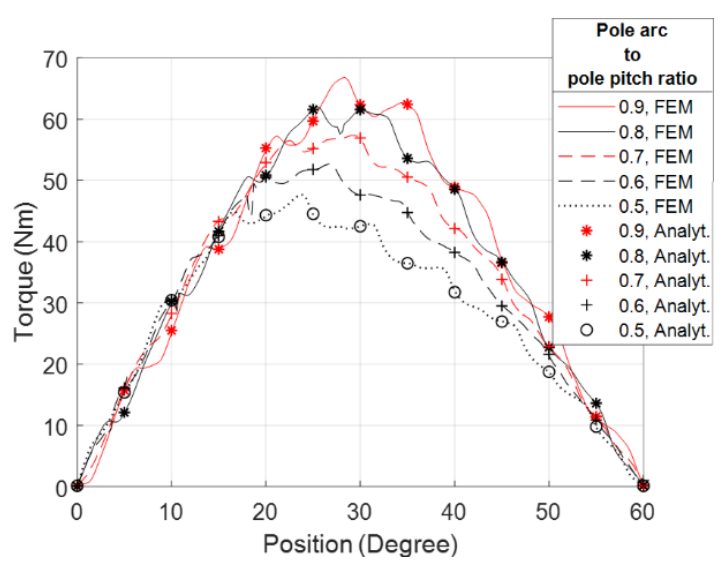

Fig. 7. The comparison of outer torque with respect to slot opening with the same slot length

increases when the saliency becomes significant. The overall torque is calculated by summation of the reluctance torque component and conventional magnetic torque. The suggested analytical method for reluctance magnetic gear gives the overall torque at present.

\section{B. Cage Rotor Bar}

In this model a solid copper bar is added in the inner rotor to support the gearbox when slip occurs at overload conditions. One of the drawbacks of magnetic gearboxes is the slip case where the gearbox cannot produce sufficient torque, so that the gearbox goes to slip region and needs time to synchronize again. The cage bars are useful as they work on the induction motor principle to provide a source torque to rotate the gear.

Magnetic gearboxes transfer the torque without any slip ratio between inner and outer coupling. However; the torque angle recognizes sudden changes and keeps constant depending on the load which is applied in the gearbox. This sudden change of load makes the gearbox produce an angle between outer rotor and inner rotor. Therefore, the coupling will shift depending on the load torque.

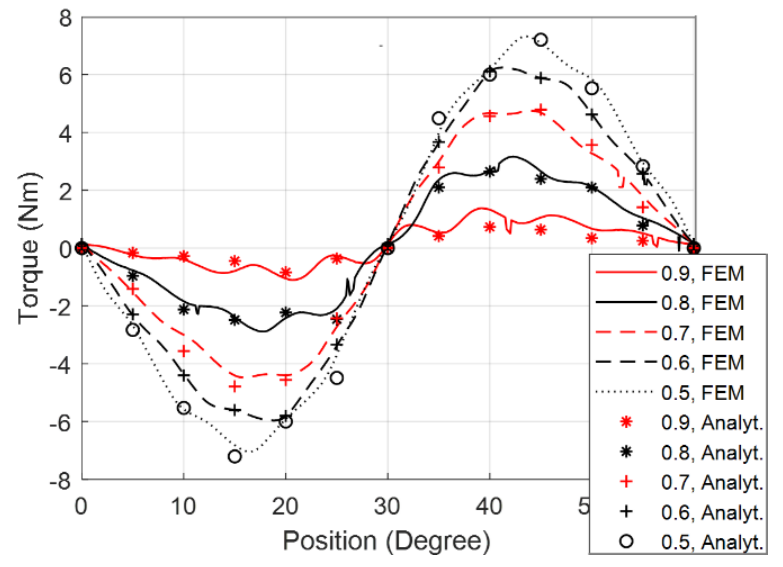

Fig. 8. The output torque due to pure reluctance torque

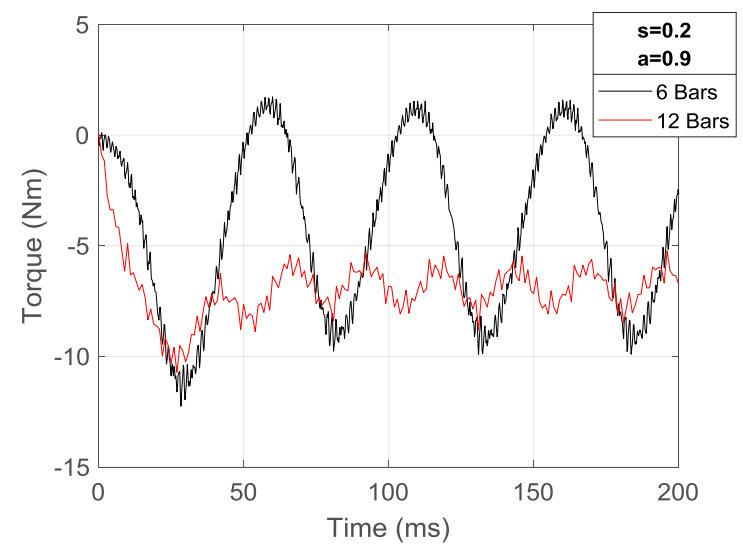

Fig. 9. Rotor cage torque ripple at 0.2 slip

The change of angle makes the cage rotor bars produce eddy current and helps to synchronize the relative speed between inner and outer rotors. As permeability of the copper is very close to permeability of free space, it is also important to investigate reluctance torque in cage rotor bar effects.

Fig. 10 shows the radial component of flux density distribution with respect to slip. In this analysis, the pole-arc to pole-pitch ratio is kept 0.9 with six rotor bars.

The torque production due to cage rotor bars is shown in Fig. 11. The figure presents the torque components on inner rotor and outer rotor for two cases, 6 bars and 12 bars. Fig. 9 shows the FE results of the torque ripple on the outer rotor generated by the cage during a step of the slip from zero to 0.2 at time $0 \mathrm{~ms}$. The results show also the steady state contribution of the cage torque for $0.2 \mathrm{slip}$.

The difference in airgap flux density distribution is because of the cage rotor. The difference between inner rotor and outer rotor speed produces eddy current in the rotor bars. Thus, the impact of the eddy currents drives the rotor into synchronism. The eddy currents in one of the rotor bars with respect to slip is shown in Fig. 12. 


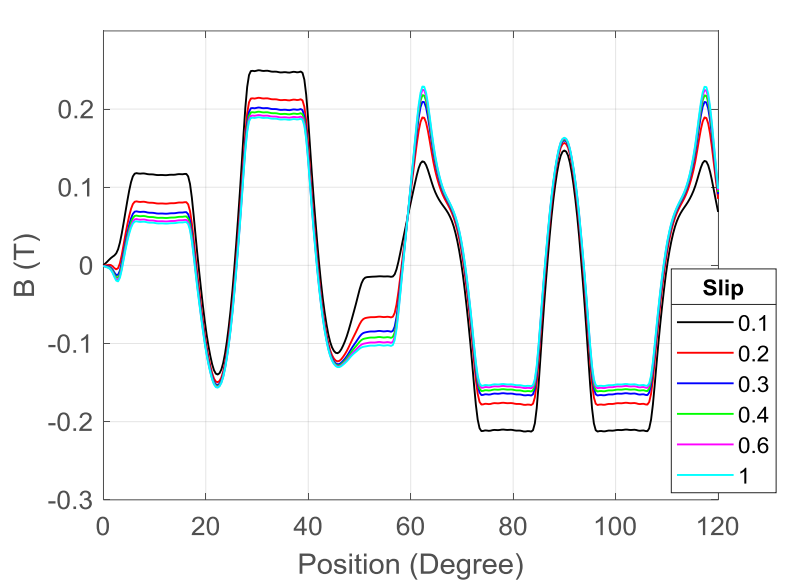

Fig. 10. Radial flux density distribution in the inner airgap with respect to slip

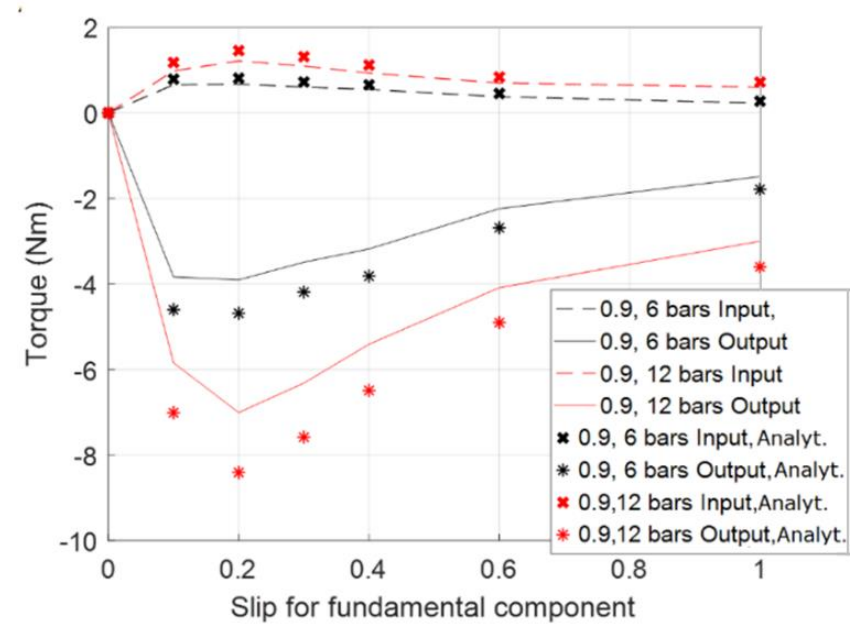

Fig. 11. Torque slip curve of inner rotor

The modulated magnetic field from the stationary part and the modulating ring induces a contrary magnetic field in the cage rotor bars. Thus, the rotor starts to synchronize the speed and removes the slip. The analytical torque results in Fig. 11 are obtained by considering the fundamental component of the magnetic field. The other harmonics can be considered separately to obtain the overall torque. Similarly, the current distribution shown in Fig.12 is due to fundamental component only. The existence of the rotor bars gives advantages specifically at overload condition. It is well known that MGs have contactless torque transmission in which slip is inevitable at overload conditions.

\section{Results Comparison}

In this section, the comparison of the significant results is presented. The gearbox size is the same for the conventional MG, reluctance MG and cage rotor MG. Fig. 13 presents the comparison of the conventional MG and reluctance MG with the same pole-arc to pole-pitch ratio, 0.5 .

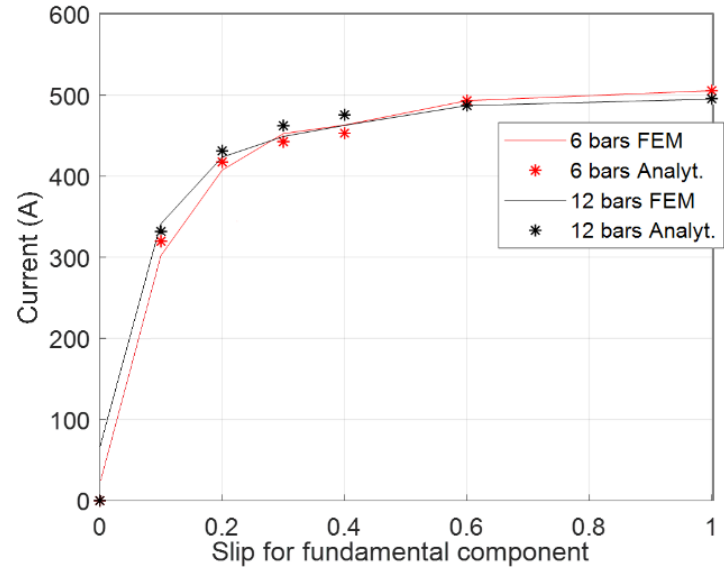

Fig. 12. Radial flux density distribution in the inner airgap with respect to slip

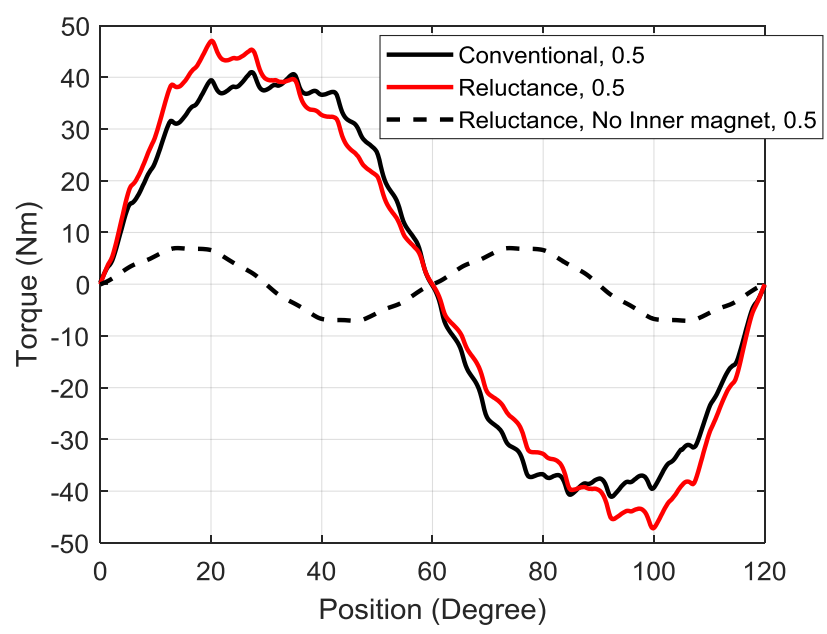

Fig. 13. The static output torque in conventional and reluctance gears

The reluctance MG produces a reluctance torque due to the salient structure of the rotor. The reluctance torque can be observed in Fig. 13. Fig. 14 illustrates the cage rotor contribution on the overall torque transmission. The torque contribution is assumed with a slip of 0.2 , which means that when the gearbox is over loaded, torque will be produced. The modulated magnetic field from the stationary part and the modulating ring induces a contrary magnetic field in the cage rotor bars. Thus, the rotor starts to synchronize the speed and removes the slip.

Fig. 15 illustrates the output torque in detail. It is visible that the cage rotor MG produces more torque than conventional MG. In the working principle of the MGs, the magnetic field produced by the outer permanent magnets are modulated by the modulating ring. The modulated field rotates synchronous with the magnetic field produced by the inner permanent magnets. In case of overload condition, both fields notice a phase shift. This causes an oscillation on the speed and torque. Thus, the cage rotor bars help to synchronize both fields under overload condition. 


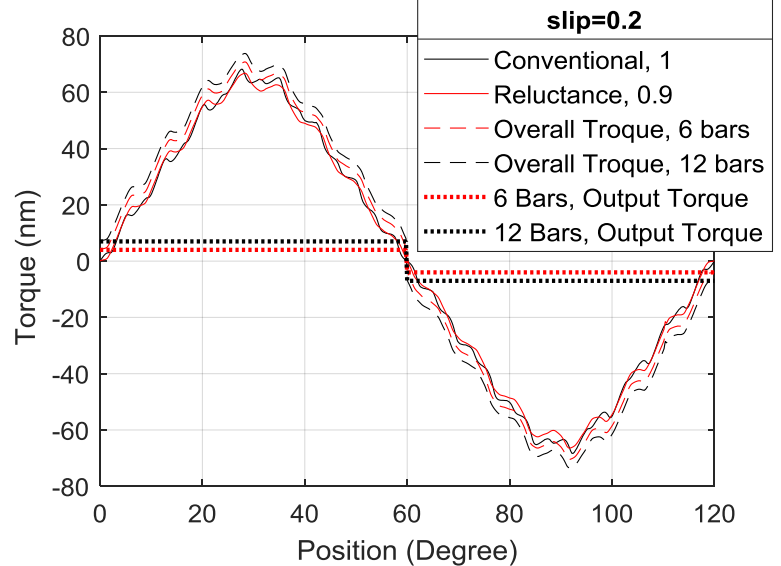

Fig. 14. The torque comparison on the outer rotor between the conventional MG and cage rotor MG

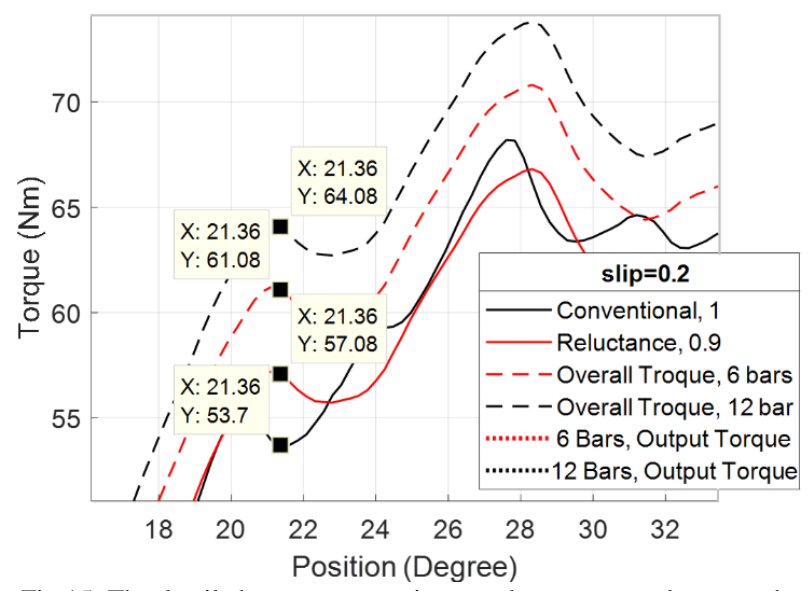

Fig.15: The detailed torque comparison on the outer rotor between the conventional MG and cage rotor MG

Fig. 16 and 17 display the current density distribution in the rotor bar in the 6-bar and 12-bar MGs, respectively. The current density values are obtained under locked rotor conditions and with the fundamental harmonic component, $\mathrm{s}=1$ and $\mathrm{n}=1$. It can be observed that the current density is larger near the surface of the copper bar and decreases through the end of the bar. The results obtained by the $2 \mathrm{D}$ analytical method, showing a good accordance with the numerical results. The 6-bar rotor configuration has significantly higher current density than the 12-bar configuration. The results suggest using the 12-bar configuration to reduce the losses and the thermal stress on the inner rotor.

Analysis of the computation time for various methods has been carried out for FEA and analytical approaches. In particular, for both the approached, two cases have been taken into considerations for the comparison. The first approach for the analytical method selects the fundamental harmonics of the permanent magnet excitation in the inner and outer PM regions for the conventional and reluctance magnetic gear. Method 2 considers all harmonics including some system harmonics that do not have contribution in the PM excitation.

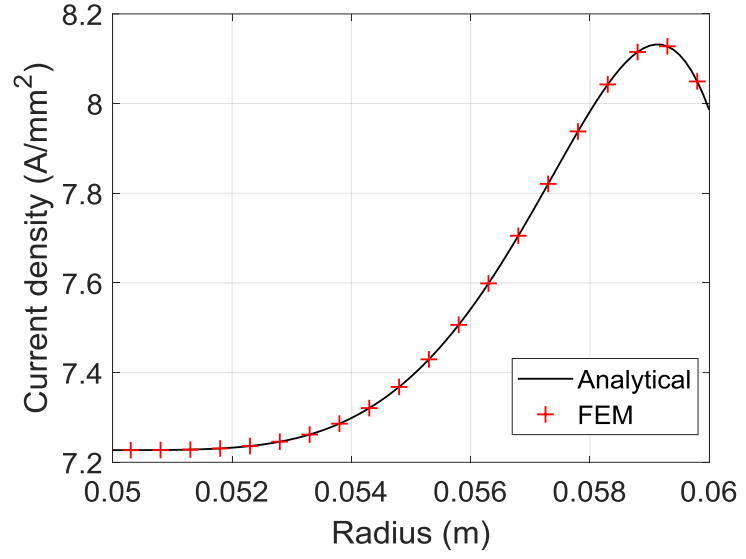

Fig. 16. Current density distribution in the 1 st rotor bar at locked rotor condition, $\mathrm{s}=1$ and $\mathrm{n}=1$, the 6 -bar MG

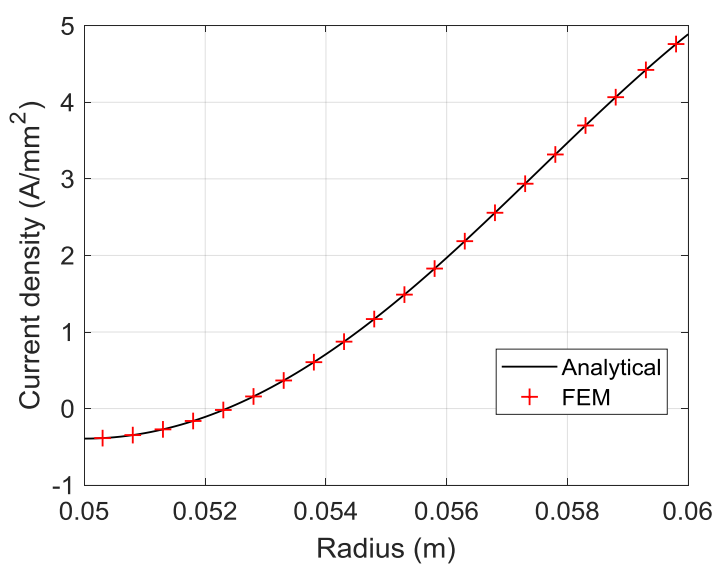

Fig. 17. Current density distribution in the 1 st rotor bar at locked rotor condition, $\mathrm{s}=1$ and $\mathrm{n}=1$, the 12-bar MG

The mesh size for FEA in method 1 has been selected 0.72 $\mathrm{mm}$ in the airgaps, $0.9 \mathrm{~mm}$ in the PM regions, and $3 \mathrm{~mm}$ for all iron core materials; whereas, method 2 has a mesh size one third of method 1.

The computation times for the proposed analytical method and FEA are listed in table II. The analytical method for the cage rotor MGs shows a substantial reduction in the solution time, with solution speed almost 72 times lower than the lowmesh size FEA and almost 8 times lower than high-mesh size FEA. The results are obtained by solving and simulating the torque result when $s=0.2$. FEA has a significantly higher computation time due to the requirement for a transient solution. In order to see the steady state torque results, FEA needs a significant transient time while the analytical method directly shows the transient results. It should also be noted that the analytical method for the cage rotor MG considers one field harmonic at a time in the permanent magnet excitation. However, all other regions have all relevant harmonics. 
TABLE II

COMPUTATION TIME COMPARISON

\begin{tabular}{|c|c|c|c|c|}
\hline \multirow{2}{*}{ MG Type } & Analytical & Analytical & FEM & FEM \\
\hline & Method 1 & Method 2 & Method 1 & Method 2 \\
\hline Conv. & $95 \mathrm{sec}$. & $935 \mathrm{sec}$. & $187 \mathrm{sec}$. & $4752 \mathrm{sec}$. \\
\hline 6-slot Rel. & $146 \mathrm{sec}$. & $1230 \mathrm{sec}$. & $396 \mathrm{sec}$. & $5602 \mathrm{sec}$. \\
\hline 12-slot Rel. & $393 \mathrm{sec}$. & $1508 \mathrm{sec}$. & $480 \mathrm{sec}$. & $6400 \mathrm{sec}$. \\
\hline 6-bar MG & $83 \mathrm{sec}$. & $279 \mathrm{sec}$. & $2220 \mathrm{sec}$. & $20098 \mathrm{sec}$ \\
\hline 12-bar MG & $110 \mathrm{sec}$. & $410 \mathrm{sec}$. & 2442 sec. & $21818 \mathrm{sec}$. \\
\hline
\end{tabular}

The final gearbox performance is given in detail in table III. The peak torque is evaluated considering 0.2 slip for the CRMGs; while the ripple is determined at zero slip (note that at 0.2 slip the torque ripple would be higher for 6 cage bars, as shown in Fig. 9). The eddy currents and core losses of the proposed reluctance and cage rotor bars are slightly lower than the conventional MG. However, the cage rotor MGs have an additional loss due to slip losses in the copper bars. The 6-bar MG has $14 \mathrm{~W}$ copper loss at peak torque operation, while the 12-bar configuration has $34 \mathrm{~W}$.

Although the efficiency of the cage rotor MGs suffers slightly due to copper losses, the maximum torque shows a higher value by comparison with the conventional and reluctance MGs.

TABLE III GEARBOX PERFORMANCE

\begin{tabular}{|c|c|c|c|c|c|}
\hline Item & Conv. & $\begin{array}{c}\text { 6-slot } \\
\text { rel. }\end{array}$ & $\begin{array}{l}\text { 12-slot } \\
\text { rel. }\end{array}$ & $\begin{array}{c}\text { 6-bar } \\
\text { MG } \\
(\mathrm{s}=0.2)\end{array}$ & $\begin{array}{c}\text { 12-bar } \\
\text { MG } \\
(\mathrm{s}=0.2)\end{array}$ \\
\hline Peak torque & $67.5 \mathrm{Nm}$ & $66.2 \mathrm{Nm}$ & $65 \mathrm{Nm}$ & $71 \mathrm{Nm}$ & $\overline{74 \mathrm{Nm}}$ \\
\hline $\begin{array}{l}\text { Torque } \\
\text { ripple }\end{array}$ & $5.8 \mathrm{Nm}$ & $6.2 \mathrm{Nm}$ & $8.8 \mathrm{Nm}$ & $6 \mathrm{Nm}$ & $7.6 \mathrm{Nm}$ \\
\hline Core loss & $13.13 \mathrm{~W}$ & $12.03 \mathrm{~W}$ & $11.46 \mathrm{~W}$ & $11.2 \mathrm{~W}$ & $10.56 \mathrm{~W}$ \\
\hline $\begin{array}{c}\text { Eddy } \\
\text { current loss }\end{array}$ & $68 \mathrm{~W}$ & $60.4 \mathrm{~W}$ & $58 \mathrm{~W}$ & $60.2 \mathrm{~W}$ & $61 \mathrm{~W}$ \\
\hline $\begin{array}{l}\text { Copper } \\
\text { Loss }\end{array}$ & 0 & 0 & 0 & $14 \mathrm{~W}$ & $34 \mathrm{~W}$ \\
\hline Efficiency & $94 \%$ & $94.3 \%$ & $94.44 \%$ & $93.7 \%$ & $92.6 \%$ \\
\hline $\begin{array}{l}\text { Torque } \\
\text { density }\end{array}$ & $\begin{array}{l}35.96 \\
\mathrm{Nm} / \mathrm{L}\end{array}$ & $\begin{array}{l}35.27 \\
\mathrm{Nm} / \mathrm{L}\end{array}$ & $\begin{array}{l}34.63 \\
\mathrm{Nm} / \mathrm{L}\end{array}$ & $\begin{array}{c}37.9 \\
\mathrm{Nm} / \mathrm{L}\end{array}$ & $\begin{array}{l}39.42 \\
\mathrm{Nm} / \mathrm{L}\end{array}$ \\
\hline
\end{tabular}

\section{CONCLUSION}

In this paper, an analytical model has been introduced to investigate non-conventional magnetic gearboxes in more design aspects. The first model has been developed to compute the reluctance torque by the influence of a saliency structure of the inner rotor. The second model has a cage rotor bar subdomain. The induced currents in the solid copper bars in the inner rotor of the MG have been analytically calculated and the effects of the induced currents have been pointed out. The methods have been validated by linear finite element analysis. The analytical models allow sufficient reduction of computational time by comparison with FEA. The results show that a considerable amount of reluctance torque can be produced by changing the design of the inner rotor parameters. In addition, the cage rotor bars proved the torque contribution at overload condition.

Analytical results have good agreement with the results obtained by FEA. Therefore, the proposed analytical models can be used as a design tool for the design of different typologies of magnetic gearboxes.

\section{REFERENCES}

[1] K. Atallah, J. Rens, S. Mezani, and D. Howe, "A novel pseudo direct drive brushless permanent magnet machine," IEEE Trans. Magn., vol. 44, no. 11 , pp. 4349-4352, Nov. 2008

[2] E. Devillers, J. Le Besnerais, T. Lubin, M. Hecquet, J.-P. Lecointe, "An improved 2-D subdomain model of squirrel-cage induction machine including winding and slotting harmonics at steady state", IEEE Trans. Magn., vol. 54, no. 2, Feb. 2018.

[3] T. Lubin, S. Mezani, and A. Rezzoug, "Analytic calculation of eddy currents in the slots of electrical machines: Application to cage rotor induction motors," IEEE Trans. Magn., vol. 47, no. 11, pp. 4650-4659, Nov. 2011.

[4] S.Williamson, L. H. Lim, and A. C. Smith, "Transient analysis of cage induction motor using finite-elements," IEEE Trans. Magn., vol. 26, No. 2, pp. 941-944, Mar. 1990.

[5] S. Mezani, B. Laporte, and N. Takorabet, "Saturation and space harmonics in the complex finite element computation of induction machine," IEEE Trans. Magn., vol. 41, no. 5, pp. 1460-1463, May. 2005.

[6] M. Perry and T. Jones, "Eddy current induction in a solid conducting cylinder with a transverse magnetic field," IEEE Trans. Magn., vol. MAG-14, no. 4, pp. 227-232, Jul. 1978.

[7] T. Lubin, S. Mezani, and A. Rezzoug, "Analytical computation of the magnetic field distribution in a magnetic gear," IEEE Transactions on Magnetic, vol. 46, no. 7, pp. 2611-2621, 2010.

[8] L. Jing and Y. Zhang, "Exact analytical method for magnetic field computation in the concentric magnetic gear with Halbach permanentmagnet arrays," in Proc. IEEE Int. Conf. Appl. Superconductivity Electromagnetic Devices, Oct. 2013, pp. 343-346.

[9] H. Y. Li, H. Hao, M. J. Jin, et al., "Analytical Calculation of Magnetic Field Distribution in Magnetic Gears with Consequent-Pole Rotors by Subdomain Method," in Proc. of Conf. on Vehicle Power and Propulsion Conference (VPPC 2016), 2016, pp. 1-6.

[10] T. Lubin, S. Mezani, A. Rezzoug, "Development of a 2-D Analytical Model for the Electromagnetic Computation of Axial-Field Magnetic Gears", IEEE Trans. Magn., vol. 49, no. 11, pp. 5507-5521, Nov. 2013.

[11] H. Y. Li, H. Hao, M. J. Jin, et al., "Analytical Calculation of Magnetic Field Distribution in Magnetic Gears with Consequent-Pole Rotors by Subdomain Method," in Proc. of Conf. on Vehicle Power and Propulsion Conference (VPPC 2016), 2016, pp. 1-6.

[12] K. Aiso, K. Akatsu and Y. Aoyama, "A Novel Reluctance Magnetic Gear for High-Speed Motor," in IEEE Transactions on Industry Applications, vol. 55, no. 3, pp. 2690-2699, May-June 2019

[13] A. Rahideh, A.A. Vahaj, M. Mardaneh et al., "Two-dimensional analytical investigation of the parameters and the effects of magnetisation patterns on the performance of coaxial magnetic gears", IET Electr. Syst. Transp., vol. 7, no. 3, pp. 230-245, 2017.

[14] M. Desvaux, B. Traullé, R. L. G. Latimier, S. Sire, B. Multon, H. B. Ahmed, "Computation time analysis of the magnetic gear analytical model", IEEE Trans. Magn., vol. 53, no. 5, May 2017.

[15] Y. Akcay, T. Cox, and A. Costabeber, "Improving magnetic gear overload torque with cage rotor bars," in 2017 20th International Conference on Electrical Machines and Systems (ICEMS), Aug 2017, pp. $1-5$. 